\title{
Le discours diplomatique dans la correspondance franco-allemande 1871-1914
}

The diplomatic discourse in the correspondence between France and Germany from 1870 to 1914

\section{Sivan Cohen-Wiesenfeld}

\section{(2) OpenEdition}

\section{Journals}

Édition électronique

URL : http://journals.openedition.org/aad/413

DOI : $10.4000 /$ aad. 413

ISSN : 1565-8961

Éditeur

Université de Tel-Aviv

Référence électronique

Sivan Cohen-Wiesenfeld, «Le discours diplomatique dans la correspondance franco-allemande 1871-1914 », Argumentation et Analyse du Discours [En ligne], 1 | 2008, mis en ligne le 18 septembre 2008, consulté le 10 décembre 2020. URL : http://journals.openedition.org/aad/413 ; DOI : https:// doi.org/10.4000/aad.413

Ce document a été généré automatiquement le 10 décembre 2020.

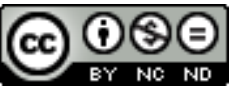

Argumentation \& analyse du discours est mis à disposition selon les termes de la licence Creative Commons Attribution - Pas d'Utilisation Commerciale - Pas de Modification 4.0 International. 


\section{Le discours diplomatique dans la correspondance franco-allemande 1871-1914}

The diplomatic discourse in the correspondence between France and Germany

from 1870 to 1914

Sivan Cohen-Wiesenfeld

\section{1. État de la recherche}

1 Le domaine de la diplomatie a fait l'objet d'investigations dans les champs les plus divers : études philosophiques et politiques à tendance morale, ouvrages de diplomates et de juristes, recherches en histoire des relations internationales, diplomatique ${ }^{1}$, études sur la négociation et, plus récemment, tentatives d'études sémiotiques, et même ébauche d'analyses dans le domaine de la pragmatique linguistique (Kurbalija and Slavick 2001; Pascual 2004). Cependant, le discours diplomatique en tant que tel n'a jusqu'à présent pratiquement pas été étudié par les sciences du langage ${ }^{2}$. La présente étude est prélevée sur un travail de plus grande envergure (une thèse de doctorat) qui se propose de combler cette lacune ${ }^{3}$. Il tente en effet de mettre en lumière le fonctionnement discursif de l'échange diplomatique à l'aide des méthodes élaborées dans les domaines de l'analyse du discours, de la pragmatique et de l'analyse argumentative. Ce faisant, il prend en compte le contexte spatio-temporel de l'interaction, et pose l'hypothèse que l'analyse de la rhétorique épistolaire peut contribuer à la compréhension des relations franco-allemandes de l'époque.

\section{Le corpus}

2 Le corpus consiste en un ensemble de lettres tirées des quarante tomes d'archives diplomatiques, publiées entre 1929 et 1958 par la commission de publication des 
documents relatifs aux origines de la guerre de 1914 du Ministère français des Affaires étrangères. Cette publication avait eu lieu suite à une polémique, au lendemain de la guerre de 1914, sur la responsabilité de la guerre. Les alliés ayant accusé l'Allemagne de porter l'entière responsabilité $\mathrm{du}$ conflit, celle-ci avait rapidement entrepris un immense effort d'historiographie qui avait abouti, dès 1922 et jusqu'en 1926, à la publication accélérée des quarante volumes de la série La grande politique des cabinets européens. Suite à quoi les alliés eux-mêmes ouvrirent leurs archives, sans tenir compte de la loi des cinquante ans, pour les publier également sous forme de recueil. Les quarante tomes de documents diplomatiques français font donc le pendant avec les quarante tomes allemands publiés auparavant. Ces documents recouvrent l'ensemble des relations internationales entre la fin de la guerre franco-allemande de 1870 jusqu'à la première guerre mondiale. Mon investigation s'est limitée à la partie de la correspondance qui concerne les relations entre la France et l'Allemagne, en prenant appui plus spécialement sur certains points de cette longue période, comme par exemple l'immédiat après-guerre, l'immédiat avant-guerre, les deux crises francoallemandes de la période bismarckienne ou les deux crises marocaines du début du siècle.

\section{Genres de correspondance}

\subsection{La dépêche et la note}

3 Ce corpus, rassemblé par des historiens, "mélange", dans sa présentation chronologique, des types d'échanges épistolaires très différents: d'une part les rapports envoyés par les ambassadeurs à leur ministre, et les instructions de ceux-cidocuments internes au ministère-, et d'autre part, la correspondance entreÉtats, c'està-dire les différentes formes de lettres, notes etc. échangées, par exemple, entre l'ambassadeur de France en Allemagne et le secrétaire d'État allemand aux Affaires étrangères, ou entre l'ambassadeur d'Allemagne à Paris et le ministre français des Affaires étrangères. Sur le plan de l'archive historique, tous ces documents sont essentiellement considérés comme des témoignages ou des preuves, entre lesquels les historiens font des recoupements dans le but d'attester, de confirmer, d'infirmer ou de modifier des hypothèses. Il s'agit pourtant de documents foncièrement différents, correspondant à des formes d'interaction distinctes entre les différents acteurs, qui se situent dans le cadre de ce que l'on appelle la démarche diplomatique et répondent aux différentes fonctions des diplomates. C'est l'ensemble de ces documents (rapports internes des ambassadeurs et correspondance entre États) que l'on désigne sous le terme très général de « correspondance diplomatique ».

4 Une première conclusion s'impose donc: sur le plan d'une analyse discursive, il est fondamental de distinguer nettement entre ces deux types de correspondance. On ne peut parler de la correspondance diplomatique de façon globale; on est obligé de se référer expressément à deux genres ou sous-genres de cette correspondance, répondant à deux pratiques socio-discursives différentes, qui possèdent des objectifs indépendants. 


\subsection{Contraintes génériques}

5 Cette distinction est d'ailleurs connue dans les milieux diplomatiques, où l'on distingue nettement entre "les dépêches et les notes" (dépêches diplomatiques est le nom générique de tous les courriers, lettres ou télégrammes échangés au sein du ministère ; notes diplomatiques est le nom générique de toutes les formes de correspondance entre les États, qui s'étendent de la «note verbale " non signée adressée à la suite d'une conversation et en reprenant les termes, à la lettre diplomatique signée, en passant par toutes les formes de notes et mémorandums ou mémoires à la troisième personne). Les règles concernant ces deux types de correspondance ont été établies au cours des siècles et rassemblées d'abord dans des ouvrages de diplomates. Le plus ancien et le plus reconnu est celui du diplomate et homme de lettres François de Callières (1717), à la fin du règne de Louis XIV, De la manière de négocier avec les souverains, qui est devenu aujourd'hui un des classiques internationaux de la négociation (réédité en 2000 aux États-Unis). Il a été suivi par des manuels de diplomatie et de droit diplomatique. On retrouve jusque dans des ouvrages récents les mêmes règles, actualisées. Elles se traduisent par un certain nombre de contraintes génériques. Le rapport, par exemple, bien qu'il se présente sous forme de lettre, doit être complet, véridique, ne pas chercher à persuader mais à informer, et surtout «ne pas prétendre éclairer le gouvernement dont il attend des ordres » (objectif, informatif et non argumentatif). Il doit correspondre à l'ethos de l'honnête homme, qui est capable de parler de tout sans se piquer de rien (Maximes de La Rochefoucauld, 1665), et pour lequel, entre autre, « Le moi est haïssable » (Pensées de Pascal, 1660).

6 Les notes que les gouvernements étrangers échangent entre eux sont, au contraire, des textes argumentatifs qui ont pour but de convaincre la partie adverse et d'emporter son consentement. Mais elles doivent le faire sous une forme impersonnelle et distante ( $d$ 'où l'emploi fréquent de la troisième personne), en respectant un ensemble de règles strictes concernant la forme des documents, la terminologie et les formules de courtoisie qui relèvent du protocole, un style qui comprend la clarté de l'expression, l'emploi de termes appropriés ne laissant pas de place à l'équivoque), et la forme de l'argumentation : construction logique, rigueur du raisonnement; pas de sous-entendus, de phrases obscures, de contradictions, de conclusions ne découlant pas des prémisses, d'arguments mineurs mêlés aux arguments importants. De plus, elle se caractérise par le respect indispensable des règles de la courtoisie internationale, dont le rôle est notamment de réglementer l'expression de la subjectivité. Pas d'insultes ni d'injures, des expressions "adoucies", dans le cas de la protestation des euphémismes et des périphrases permettant de contourner les expressions trop crues. Ces règles sont contraignantes, puisque leur omission peut aboutir au rejet de la note (renvoi à l'expéditeur).

\subsection{Respect/contournement des contraintes génériques}

7 Or, si la note respecte relativement bien ses contraintes génériques, le rapport par contre, les enfreint fréquemment, soit directement, soit indirectement.

8 Pour illustrer la rhétorique de la note diplomatique, texte présentant une dimension à la fois fortement argumentative et très formaliste, nous prendrons un exemple 
extrême, la dernière lettre adressée par l'ambassadeur d'Allemagne à Paris au président de la République française avant la guerre de 1914 :

Paris, 3 août 1914

Monsieur le Président,

Les autorités administratives et militaires allemandes ont constaté un certain nombre d'actes d'hostilité caractérisée commis sur le territoire allemand par des aviateurs militaires français. Plusieurs de ces derniers ont manifestement violé la neutralité de la Belgique, survolant le territoire de ce pays. L'un a essayé de détruire des constructions près de Wesel, d'autres ont été aperçus sur la région de l'Eiffel, un autre a jeté des bombes sur le chemin de fer près de Karlsruhe et de Nuremberg.

Je suis chargé et j'ai l'honneur de faire connaitre à Votre Excellence qu'en présence de ces agressions, l'Empire allemand se considère en état de guerre avec la France $\mathrm{du}$ fait de cette dernière puissance.

[...] Ma mission diplomatique ayant ainsi pris fin, il ne me reste plus qu'a prier Votre Excellence de vouloir bien me munir de mes passeports et de prendre les mesures qu'Elle jugera utile pour assurer mon retour en Allemagne avec le personnel de l'Ambassade [...]

Veuillez agréer, Monsieur le Président, l'expression de ma très haute considération. Signé : Schoen

9 Cette lettre, qui rompt avec la tradition classique des longues déclarations de guerre motivées en détail, et dont le caractère manipulateur a été relevé par les historiens, reste cependant conforme aux codes génériques de la correspondance interétatique. Malgré l'emploi de la première personne, due à la forme épistolaire et à la nécessité pour l'ambassadeur de récupérer ses passeports diplomatiques, garants de son immunité, le locuteur parle au nom d'instances nationales, officielles et impersonnelles ("Les autorités administratives et militaires allemandes», "l'Empire allemand») et désigne son destinataire de la même façon ("des aviateurs militaires français », «la France »). Les dénominatifs et les titres protocolaires sont scrupuleusement respectés ("Monsieur le Président», "Votre Excellence », «Elle»), de même que la formule de courtoisie finale. L'argumentation, qui aboutit rapidement à la culpabilité de la France dans le déclenchement de la guerre, se fonde sur des faits "constatés» et "manifestes", les précisions géographiques parant grossièrement la nébulosité des motifs («L'un a essayé... », « d'autres ont été aperçus... ») qui justifient les appellations «d'actes d'hostilité caractérisée » et "d'agression », ou l'accusation de violation de la neutralité du territoire belge. La distance énonciative exacerbée, accentuée par le constant rappel des qualificatifs nationaux (« les autorités allemandes», le «territoire allemand», les «aviateurs militaires français » etc.) prépare le terrain, sur le plan énonciatif, à la rupture effective des relations diplomatiques dont la responsabilité est rejetée sur l'instance énonciataire ( «l'Empire allemand se considère en état de guerre avec la France du fait de cette dernière puissance»). La brièveté et la formalité du langage officiel a ici été utilisée pour bloquer définitivement la situation argumentative et légitimer le recours à la force. La note diplomatique est donc un genre de texte clairement argumentatif, présentant un point de vue, tout en respectant strictement les modalités formelles, quelle que soit la gravité de la situation, et ce pour protéger les règles du jeu entre les nations. Ces contraintes elles-mêmes sont susceptibles de faire l'objet, comme c'est le cas ici, d'une utilisation à des fins déterminées.

Le rapport, par contre, enfreint souvent ses contraintes génériques, généralement par des moyens détournés. Loin d'être un simple "commis voyageur" chargé de transmettre des messages de son gouvernement ou d'en appliquer les instructions, 
l'ambassadeur de la fin du XIXe siècle possède un rôle central dans la conduite de la diplomatie bilatérale secrète qui caractérise cette époque. Les dépêches par lesquelles il rend compte à son ministre de ses entretiens avec ses interlocuteurs étrangers reflètent l'ambiguïté de ce statut, limité institutionnellement, mais conséquent sur le terrain. L'émergence du sujet, qui est inévitable, ne peut s'y exprimer ouvertement. Ses prises de position, le cas échéant, ne peuvent se faire de façon directe, et doivent "contourner" les contraintes génériques. De même la présentation de soi de l'ambassadeur, conforme à celle de l'honnête homme, doit jongler entre le tabou sur l'auto-compliment et la nécessité de maintenir un ethos de compétence et de faire acte de qualités qui justifient sa position. Cette « rhétorique de l'indirection » se traduit par l'utilisation de diverses stratégies énonciatives.

11 Le ton général des rapports des diplomates à leur ministre dans ce corpus est proche, dans l'ensemble, de celui de la dépêche citée ci-dessous :

Voici, Monsieur le Ministre, le résumé de la conversation qui a eu lieu ce matin entre nous et Monsieur Herzog [...]

La France, a dit ce commissaire, désire que [...]

Les confidences que nous a fait le délégué du prince de Bismarck sont-elles préméditées, sérieuses, et pouvons-nous entrer dans la voie qui nous est ouverte? L'attitude même de M. Herzog, ce que nous savons des habitudes de la haute administration prussienne aurait suffi pour nous édifier à cet égard, si, comme nous vous le disions plus haut, M. le comte d'Arnim, sous la même forme d'insinuation toute personnelle, quoique en termes moins couverts, ne nous avait, de son côté, fait clairement comprendre que, sur le terrain des compensations indirectes, les demandes dont nous prendrions l'initiative trouveraient à Berlin un accueil favorable.

Permettez-nous, Monsieur le Ministre, d'ajouter, en terminant, que nous croyons à la sincérité des aveux que l'on a reçu l'ordre de nous faire ; mais, jusqu'à plus ample informé, nous sommes moins convaincus de l'étendue des avantages matériels qu'on serait disposé à nous concéder à Berlin. Les précédentes négociations auxquelles nous avons eu l'honneur d'être associés ne nous autorisent que trop à craindre, qu'en échange de sacrifice facile à chiffrer, on se croit dégagé envers nous, par la concession de ce qu'au fond on est dès aujourd'hui décidé à faire [...]

[...] Sans songer à influencer les résolutions du gouvernement, nous avons cru de notre devoir, Monsieur le Ministre, de vous faire part des impressions que nous avons pu recueillir à cet égard et d'éveiller votre attention sur l'une des faces de la question qui vient d'être soulevée ici [...]

(Dépêche du 14 juin 1871 des plénipotentiaires de France à Francfort au ministre des Affaires étrangères Jules Favre).

Le rapport débute par le compte rendu d'un entretien, soit au discours direct, soit, comme c'est le cas ici, à la forme indirecte, suivi d'une partie interprétative et délibérative. Le point de vue de l'agent, représentant de son gouvernement et négociant en son nom, ne peut émerger que prudemment, s'entourant d'un luxe de " précautions énonciatives " ( Permettez-nous, Monsieur le Ministre...", « Sans songer à influencer les résolutions du gouvernement ", "éveiller votre attention » etc.). Le compte rendu du diplomate reflète, certes, la rhétorique particulière du processus même de négociation (comme le fait de se livrer à des « confidences préméditées » ou sérieuses, ou de faire "clairement comprendre " sous forme d'insinuation), mais il s'agit aussi et avant tout d'un rapport adressé dans un cadre institutionnel précis. L' ethos de compétence est également posé de façon indirecte, esquivant le tabou sur l'auto-compliment (« Les précédentes négociations auxquelles nous avons eu l'honneur d'être associés ne nous autorisent que trop... »). 
13 La «transgression ouverte » des contraintes génériques du rapport, plus rare, se remarque cependant dans le corpus, à une époque où « les grands ambassadeurs [...] [n'] ont assez de caractère et d'autorité personnelle pour devenir, dans les circonstances graves, les conseillers de leur gouvernement et prendre même parfois une allure de "mentor" " (Renouvin 1955 : 378-379). Lors de la seconde crise marocaine, par exemple, l'ambassadeur Jules Cambon, partisan de la signature d'un accord avec l'Allemagne, s'opposa à la politique de son ministre des affaires étrangères, de Selves, moins souple, qui, lui, souhaitait la réunion d'une nouvelle conférence internationale. A la suite d'une joute épistolaire au cours de laquelle la règle selon laquelle il ne doit « pas chercher à persuader mais à informer», et surtout « ne pas prétendre éclairer le gouvernement dont il attend des ordres » fut largement enfreinte, l'ambassadeur finit par avoir gain de cause ${ }^{4}$. Notons qu'au contraire, les lettres du ministre ou du Président à son agent (rapport hiérarchique inversé) sont, elles, plus libres sur le plan de l'expression, moins tenues par les contraintes de forme et laissent libre cours notamment à la construction de l'ethos ${ }^{5}$.

Il faut ajouter, enfin, qu'à partir de 1875, les Chambres délibérantes peuvent demander que leur soit transmises des pièces concernant la sécurité de l'Etat, pièces qui étaient traditionnellement confidentielles et secrètes. Pour pallier ce risque de publicité devant les chambres, on voit se développer la pratique des «lettres particulières » entre l'agent et son ministre, lettres confidentielles personnelles moins formelles que la correspondance officielle, qui restent la propriété privée de leur rédacteur ou de leur destinataire, et ne sont pas obligatoirement reversées dans les Archives du ministère. Portant en principe sur des questions personnelles, ces lettres ont eu tendance à tenir le rôle des dépêches officielles, particulièrement en période de crise. Les exemples suivants montrent un autre aspect de cette correspondance dans laquelle les énonciateurs, tout en se limitant à l'objet formel du discours, utilisent cependant des dénominatifs affectifs et des termes axiologiques, qui relèvent clairement du domaine de la subjectivité :

Faisons donc, mon cher ami, tous nos efforts pour déjouer même, s'il est possible, les desseins hostiles. En ne négligeant rien, en nous armant d'une grande et persévérante patience, en restant corrects dans tous nos actes, en nous renfermant dans nos affaires intérieures si graves déjà par elles-mêmes, nous n'aurons du moins rien à nous reprocher.

(Dépêche personnelle de l'ambassadeur Gontaut-Biron au Duc Decazes, 26 décembre 1873)

Je pense souvent à vous et je vous plains, car je comprends tout ce que votre situation doit avoir de douloureux en ce moment. Ce n'est pas que nous n'ayons pas aussi notre part d'angoisses. Les députés alsaciens du Reichstag ont écrits ou vu nos députés des départements du Nord-Est...

(Lettre particulière du 23 janvier 87 du ministre Flourens à l'ambassadeur Herbette)

En reprenant les classifications proposées par Jean-Michel Adam (1998: 46-53) et Jürgen Siess (1998: 111), j'ai donc proposé d'assimiler la note ou correspondance interétatique à la correspondance d'affaire - la plus éloignée sur l'échelle de la formalité et de la distanciation, brève et limitée à l'objet formel du discours. La dépêche ou rapport diplomatique serait par contre comparable à la lettre à enjeu relationnel, de par son dispositif d'énonciation (je/vous), la place qu'y occupent l'ethos et la construction de l'image de soi et de l'autre, et le but - sollicitation, maintien, développement, reprise d'une relation. 

reste dans la formalité et la distance, et un autre dans lequel se concilient fonction officielle et sphère privée confidentielle. Ces deux types de documents, qui sont mis sur un même plan dans le corpus historique dont ils sont tirés, présentent pourtant deux aspects différents d'un même discours institutionnel "clivé ", scindé entre des échanges discursifs confidentiels et une rhétorique officielle tournée vers l'extérieur.

\subsection{Contraintes antinomiques et tensions internes du discours}

17 Cependant, ces deux sous-genres relèvent d'un même type de discours: le discours diplomatique. Diplomates et spécialistes des sciences politiques le répètent dans tous leurs ouvrages : le discours du diplomate possède une visée spécifique et répond aux contraintes antinomiques qui pèsent sur la diplomatie : il s'agit de concilier la défense des intérêts nationaux particuliers tout en empêchant la guerre, voire en construisant la paix. La communication diplomatique est donc le fruit d'un compromis constant entre des exigences contradictoires. Cette caractéristique émerge en particulier dans les négociations. Forme pacifique de résolution des conflits internationaux, la négociation diplomatique ne cherche pas à éviter ou à ignorer les conflits d'intérêts, inhérents aux relations internationales. Elle a pour but de s'y confronter, mais même si elle ne parvient pas à concilier les vues contradictoires, elle doit cependant s'efforcer d'éviter l'échec radical de l'interaction qui marque la fin de la paix. Les conséquences d'un tel échec prennent ici des proportions d'ordre international. Ces contraintes antinomiques sont à l'origine des tensions internes qui traversent ce discours de part en part, et qui se traduisent par des "choix» discursifs et linguistiques, lesquels trouvent leur expression dans la correspondance diplomatique.

\subsection{Discours officiel et subjectivité}

La première tension est celle entre la fonction officielle et la sphère de la subjectivité. Le discours diplomatique émane de personnes au service de l'État dans l'exercice de leur charge. A ce titre, il possède les propriétés du discours officiel, formel et impersonnel, soumis à des astreintes hiérarchiques et protocolaires et régi par des codes spécifiques. Ceci est encore plus vrai quand il s'agit d'écrits. Aussi bien la correspondance entre États que les rapports internes des diplomates apparaissent comme se conformant à ces contraintes de neutralité, d'objectivité (chacun « dans son genre » : la note en conformité avec les règles du protocole international et le rapport avec celles du contexte hiérarchique). Le langage de la diplomatie se doit de rester dans le domaine de l'impartialité, la neutralité, la précision, la logique et la régularité afin d'éviter les contresens et de préserver l'harmonie des relations entre les États. Il est pourtant frappant de constater l'importance de l'élément subjectif dans un domaine où il est censé ne pas exister, ainsi que l'étrange imbrication de la sphère publique et de la sphère privée qui le caractérise La dépêche diplomatique est une forme particulière de rapport: son but essentiel reste l'information du destinataire, mais les enjeux relationnels, la construction de l'image de soi et la négociation de la relation interpersonnelle y prennent une place prépondérante, favorisée par le dispositif d'énonciation. D'autre part, la subjectivité du scripteur y émerge sans cesse sous la forme d'« impressions " (ce mot revient au moins une fois dans pratiquement chaque texte), de "sentiments ", d'interprétation du " climat général », de réflexions d'ordre 
psychologique sur les personnes, d'analyses relevant autant du domaine de la psychologie que de celui la politique pure (voir le portrait de Guillaume II par Herbette en annexe). Qu'il s'agisse de "ménager des susceptibilités», ou de "changer des sentiments ", le champ sémantique est souvent celui de l'affectif :

Pendant les fêtes... j'ai été comblé d'attentions particulières par l'Empereur, l'Impératrice et son entourage. ... Je n'ai d'ailleurs observé, pendant le cours de ces fêtes à Berlin, aucune parole, aucune manifestation dont notre susceptibilité n'aurait pu être blessée...

Je crois devoir signaler ces circonstances parce qu'il me parait important pour les rapports entre la France et l'Allemagne qu'au moment où la presse de tous les pays de l'Europe retentit d'adulations intéressées en l'honneur du vieil Empereur, il ne se produise pas chez nous quelques-unes de ces récriminations amères ou ironiques qui ont le don de blesser si profondément le sentiment national allemand.

(Télégramme personnel du 5 janvier 1886 du baron de Courcel à Freycinet) ${ }^{6}$

Rappelons encore que cette correspondance est confidentielle, et sauf mention explicite, destinée à être lue uniquement par le destinataire auquel elle est adressée. Entremêlant l'officiel et l'officieux, la dépêche diplomatique peut donc être, malgré ses contraintes génériques, traversée de part en part, directement ou indirectement, par la subjectivité de ses énonciateurs. On peut encore évoquer à ce sujet l'importance de la perception de l'autre et du jeu spéculaire des images dans les négociations internationales, constituant la toile de fond sur laquelle se déroulent les négociations, les ethnotypes jouant à cet égard un rôle particulier :

Le défaut de tact et la lourdeur des Allemands sont notoires... Le vingt-cinquième anniversaire de ces évènements douloureux m'a exposé à une série de démonstrations, sympathiques ou intéressées, qui, de la part de nature moins inconscientes, pourraient presque être taxées d'impertinence...

(Dépêche du 29/7/1895 de Jules Herbette au ministre Hanotaux)

Enfin, certains ambassadeurs, en particulier ceux qui, restés longtemps en poste, avaient acquis une grande expérience et une parfaite connaissance du terrain, ont eu le désir d'infléchir la politique de leur pays dans le sens qui leur semblait le plus adapté au bien national, en particulier lorsqu'ils avaient en face d'eux un ministre moins expérimenté, même si cela n'était pas leur rôle institutionnel. Aussi le poids spécifique de la personnalité individuelle est-il déterminant, même dans un cadre contraint.

\subsection{Un discours à double niveau}

21 La seconde tension interne se situe entre le niveau apparent et la dimension gommée $\mathrm{du}$ discours. Le but informatif $\mathrm{du}$ rapport, par exemple, constitue son «niveau apparent »; la négociation de la relation interpersonnelle de l'agent qui rapporte à son supérieur hiérarchique relève du «non-dit ».

L'emploi du discours de l'autre, et donc des différentes modalités du discours rapporté, est l'une des caractéristiques de ces deux types de correspondance. Dans les rapports bien sûr : l'emploi du discours direct dans le compte rendu de la négociation orale est une mise en scène ayant pour but de montrer l'autre "tel qu'il est vraiment", plus efficacement que par une argumentation explicite et donc discutable. Celui du discours indirect permet l'interprétation voilée de la parole de l'autre. Par ailleurs, rapporter ses propres paroles au discours direct permet de mettre en valeur son propre comportement, bien que le moi soit « haïssable». Le discours rapporté répond donc dans la dépêche à un double but, l'un visible, et l'autre « caché ». 
23 La correspondance interétatique, quant à elle, se présente comme un discours fondamentalement hétérogène, caractérisé par la présence structurante du discours antagoniste qu'elle expose pour en exhiber les faiblesseset le réfuter. Les actes de langage comme les déclarations, promesses et engagements oraux ou écrits y sont considérés comme des faits sur lesquels est fondée l'argumentation. Ils sont donc sans cesse, soit « rappelés » au partenaire, sous la forme du discours direct, pour le mettre en contradiction avec sa propre parole, comme dans le premier exemple ci-dessous, soit reconstruits au discours indirect ou au discours indirect libre, et réfutés, comme dans le second :

Me permettez-vous de vous avouer que j'ai été douloureusement impressionné en voyant passer le jour de naissance de Sa Majesté l'Empereur d'Allemagne sans que l'amnistie de nos prisonniers ait été complétée ? Vous aviez bien voulu me dire que vous nous rendriez » tous ceux qui n'auront pas été pris au coin d'un bois tirant sur nos troupes sans être revêtus d'aucun insigne militaire ». J'attends la réalisation de vos promesses. [...]

Vous m'avez dit expressément que vous vouliez la paix. Je mets la foi la plus entière en vos paroles. Veuillez m'en croire. L'un des plus sûrs moyens d'y parvenir par rapport à la France est entre les mains de Sa Majesté.

(Lettre de l'ambassadeur de France à Berlin, M. de Gontaut-Biron, à Bismarck 23/3/1872)

Vous avez bien voulu me parler dans votre lettre de l'idée d'une occupation temporaire de Rabat par les troupes françaises, une pareille occupation devant faciliter une expédition pour venir au secours des colonies à Fez. Je ne saurais cependant vous cacher qu'il est à craindre que cette mesure ne soit mal vue par l'opinion publique en Allemagne. L'occupation par la France d'un deuxième port important, à côté de celui de Casablanca, serait considérée comme une étape vers l'élimination de la convention d'Algésiras, puisque tout est calme, en ce moment, à Rabat, et que l'occupation de cette ville n'aurait qu'un but indirect.

(Note du secrétaire d'Etat allemand aux Affaires étrangères, Kiderlen-Wächter à l'ambassadeur Jules Cambon, 7 avril 1911) ${ }^{7}$

De même, la subjectivité de l'énonciateur, qui ne peut s'exprimer directement ou explicitement, doit passer par le mode implicite, créant un " effet d'objectivité ", par l'utilisation des procédés de l'effacement énonciatif, tout en ayant recours aux nombreuses ressources qu'offre le langage pour introduire dans l'énonciation des éléments dirigeant l'interprétation du lecteur. Dans l'exemple ci-dessous, l'effet d'objectivité, créé par l'usage de la troisième personne "recouvre » l'emploi d'unités lexicales auxquelles sont attachées des présupposés comme «il a cru devoir», ou de termes affectifs (« susceptibilité ») ou axiologiques (« coupables de ») :

Le gouvernement allemand a cru devoir intenter des poursuites contre un certain nombre de curés de l'ancien département de la Meurthe, placés maintenant sous sa domination, et coupables d'avoir lu en chaire, il y a environ trois mois, un mandement de Mgr l'Evêque de Nancy dont les termes ont éveillé la susceptibilité du Cabinet de Berlin.

(Dépêche du 19 novembre 1873 du ministre des affaires étrangères de Broglie au chargé d'affaires à Berlin, Sayve)

Dans l'extrait suivant, c'est cette fois un adverbe focalisateur interprétatif («malheureusement») associé à un présupposé ("tout le corps diplomatique sait que »), qui véhicule le regard du locuteur (Nolke 1993) et oriente l'interprétation du destinataire, en insistant sur l'image peu fiable et ennemie de l'interlocuteur allemand dont il convient de mettre la parole à distance. Il y a donc un jeu sur le degré d'adhésion aux déclarations de ses interlocuteurs : 
Comme à notre premier entretien, $\mathrm{M}$. de Thile me témoigna un certain étonnement feint ou sincère de l'importance que j'attachais à ce point, prétendant que après tout ces prisonniers n'étaient pas si intéressants ${ }^{8}$. Si M. de Thile était le ministre dirigeant, ses assurances me donneraient un espoir sérieux; malheureusement, tout le corps diplomatique sait que le Prince de Bismarck ne se tient nullement engagé par les paroles de M. de Thile.

(Dépêche du 25 janvier 1872 de Gontaut-Biron à Rémusat) utilise la parole de l'autre, et qu'il se fonde sur une généralisation, procédés typiques de la production de "l'effet d'objectivité ». Quant aux négociations par écrit, elles voilent par les formes protocolaires et impersonnelles un débat polémique qui constitue le fond du problème.

\section{Conversation diplomatique et préservation des faces}

La négociation à huis clos abordable à travers le corpus emprunte la forme souple et informelle de la conversation, enveloppant la phase argumentative conflictuelle inhérente à toute négociation dans les méandres de cette forme d'interaction quotidienne, voire intime. Elle laisse cependant une place prépondérante au "ménagement des faces", étant donné le statut de représentants nationaux des participants. Ceci se manifeste par le respect de l'alternance des tours de parole et du principe de réciprocité de la relation en général, aussi bien au niveau formel que des thèmes abordés, le maintien du ton cordial, la préférence pour les Face Saving Acts, nécessaire conciliation de comportements discursifs antagonistes comme la sincérité et la courtoisie, la franchise et le tact. Ce respect du Face Management s'avère particulièrement nécessaire en période de crise, le caractère extrêmement ritualisé des échanges interétatiques, oraux et épistolaires, servant alors de garde-fou contre les débordements émotionnels qui caractérisent le débat public. Il existe une similitude entre cette forme réglée et contrainte d'échanges entre États caractérisée par un ensemble de rituels, de codes verbaux, de cérémonial ayant pour but le maintien de l'équilibre de la paix internationale, particulièrement en période de crise, et la communication quotidienne modelée par un système de règles interactionnelles avec lesquelles les participants doivent composer pour permettre la préservation de l'harmonie sociale dans les conflits mineurs de la vie de tous les jours. Dans les relations interpersonnelles comme dans les relations entre États, tout est une question de sauvegarde du territoire et de la face - la sienne et celle de l'autre, ce Face management masquant souvent, sous une forme consensuelle de civilité, des enjeux moins avoués de puissance et de gloire.

Il faut cependant noter que les conversations diplomatiques en face-à-face n'apparaissent dans le corpus qu'à travers le discours rapporté du diplomate. L'analyse de la négociation doit donc tenir compte du double niveau d'interaction impliqué par la correspondance : l'entretien oral faisant l'objet du rapport, mais aussi l'interaction épistolaire entre le scripteur et son destinataire. La conversation est «représentée » par l'énonciateur à l'attention de son destinataire, qui est ici son supérieur hiérarchique. Sans chercher à " déformer la réalité ", il la lui montre en fonction de ses divers objectifs propres : assurer son ethos, prouver sa compétence, montrer comme il s'est conformé aux instructions reçues, ou bien au contraire infléchir une politique etc. 
La correspondance interétatique, par contre, qui met les deux États en "face-à-face » sans intermédiaire, montre bien cette place prépondérante du Face Management et des procédés « adoucisseurs » (Kerbrat-Orrecchioni 1992 et 1994) des actes menaçants.

Les caractéristiques que le sens commun relie de façon quasi-automatique et stéréotypée au discours diplomatique, comme l'ambiguïté ou l'ostentation, prennent donc sens si on les considère dans le cadre de la visée globale d'un genre de discours et de ses contraintes.

\section{La dimension diachronique de l'échange}

30 L'approche argumentative adoptée dans cette étude privilégie l'étude des "genres » considérés comme des dispositifs de communication socio-historiquement définis. Replacé dans son contexte socio-historique, le corpus y a été étudié non seulement dans le but d'y retrouver les règles régissant le discours diplomatique en général, mais aussi et surtout les caractéristiques du discours diplomatique entre la France et l'Allemagne au cours de la période concernée. Ainsi, l'étude du corpus par « sondes » diachronique a permis de suivre l'évolution du fonctionnement de l'interaction discursive entre les deux pays au long de la période. Polémiques et conflictuels au lendemain de la guerre de 1870 comme à la veille de celle de $1914^{\circ}$, les échanges entre les diplomates se dissocient progressivement, à partir des années 80 , du bellicisme encore de règle sur la scène publique, comme en témoignent, en particulier, les dépêches confidentielles :

Il est des passions qui ne peuvent disparaître immédiatement, dis-je à mon interlocuteur, mais, en cherchant des points de contact dans chaque affaire particulière qui se présentera successivement, von fall $z u$ fall, suivant une expression que vous employez quelquefois, nous arriverons à multiplier les occasions d'accord; il pourra naître de cette manière de procéder une sorte d'habitude et l'habitude transforme les sentiments

(Dépêche du 20 janvier 1885 du baron de Courcel, ambassadeur, au ministre Jules Ferry - Confidentiel).

31 Certains diplomates feront même figure d'agents de l'ennemi (comme ce fut le cas d'Herbette à l'époque de l'affaire Schnæbelé) pour cette raison. Cependant, on n'a jamais affaire à un véritable "dialogue franco-allemand", à vocation de communication "authentique». Même pendant les périodes de rapprochement politique, la correspondance diplomatique garde un fond de méfiance, les accords ne sont obtenus que lorsqu'il n'y a pas le choix et au vu des intérêts communs, au terme de négociations à dominante conflictuelle, et non par désir de coopération attesté par le mode d'interaction. Dans les rapports internes, les frontières posées dans le texte avec le discours de l'autre, pratiquement tout au long de la période étudiée, sont révélatrices de la distance incompressible entre les deux Etats, qui malgré les déclarations de pacifisme officiel et le désir de rapprochement de certains diplomates, ne deviendront jamais des alliés. La perception de l'autre dans les rapports, fortement négative après le conflit, évolue positivement pendant les périodes de rapprochement politique, les stéréotypes péjoratifs réapparaissant cependant au moment des crises jusqu'à celle de 1905. La nomination de Jules Cambon au poste d'ambassadeur à Berlin en 1907 marque à ce titre un changement déterminant : sa grande connaissance du terrain, combinée à un patriotisme clairvoyant et un profond désir de paix lui font envisager ses interlocuteurs de façon pondérée, permettant une vision équilibrée de la situation entre les deux pays. Parallèlement à la réhabilitation graduelle de l'image de 
l'Allemagne se fait jour une certaine autocritique, qui reste cependant dans les limites du patriotisme consensuel et apparaît uniquement dans les dépêches confidentielles, portant d'abord sur la responsabilité de la guerre de 1870 (facilitée il est vrai dans ce domaine par le changement de régime), puis s'élargissant parfois à l'ensemble de la politique et de la société françaises. Cependant, l'image d'un adversaire à la fois méfiant et peu fiable perdure à travers toutes les parties du corpus étudiées, rendant illusoires les diverses tentatives de rapprochement sur des bases d'intérêts économiques (à la période coloniale, puis en 1894-1898 et enfin en 1909-1911), impuissantes à "transformer les sentiments", selon le souhait exprimé par le baron de Courcel en 1885. Ces "sentiments" étant cristallisés autour du thème de l'Alsace-Lorraine, question « hors diplomatie $»^{10}$, point d'achoppement des relations entre les deux pays.

\section{Conclusion}

L'étude des deux aspects de la correspondance diplomatique - échanges interétatiques officiels et rapports internes - permet d'appréhender le fonctionnement d'un discours institutionnel à deux faces, dont le pivot, à la fin du XIXe, début du XXe siècle, est encore constitué par le personnage de l'ambassadeur, représentant de l'État vis-à-vis de l'étranger, et en même temps informateur de son propre gouvernement.

Les notes interétatiques gardent un caractère formel et distancié, nécessaire à la préservation de l'harmonie des relations entre les États. Elles n'en sont pas moins des textes fortement argumentatifs, cristallisant des positions antagonistes, et exprimant des points de vue subjectifs "masqués " sous des arguments rationnels. Les rapports des diplomates, contournant les contraintes liées à leur but strictement informatif en contexte hiérarchique, se présentent comme des lettres à forts enjeux relationnels, dans lesquelles la construction de l'ethos et de l'image de l'autre occupe une place prépondérante, et où la subjectivité de l'énonciateur émerge sans cesse sous différentes formes.

Le rapprochement de ces deux formes de correspondance, représentant les deux faces d'un même discours, permet, notamment, de mettre en lumière le fonctionnement des échanges diplomatiques à cette période ainsi que l'évolution de l'interaction discursive entre deux pays.

Au-delà des divergences dues à l'inscription de ces genres dans des contextes énonciatifs différents, la visée particulière de la diplomatie réalise l'unité de ce discours. Obligé de concilier des contraintes antinomiques, le discours diplomatique utilise les formes de l'indirection, de l'implicite et de l'échange ritualisé pour assurer la sauvegarde du territoire et de la «face» des nations, au sens propre comme au sens figuré. La notion de frontière, qu'il s'agisse de la délimitation entre intérieur et extérieur, propre à toute institution, ou de la frontière identitaire entre le moi communautaire et l'étranger semble ici une donnée structurante du discours. 


\section{BIBLIOGRAPHIE}

Documents diplomatiques français (1871-1914) T. 1-40. 1929. Ministère des Affaires Étrangères, Commission de publication des documents relatifs aux origines de la guerre de 1914 (Paris : Imprimerie nationale)

Die Grosse Politik der Europaïschen Kabinette (1871-1914). 1922. Sammlung der Diplomatischen Akten des Auswärtigen Amtes (Berlin : Deutsche Verlagsgesellschaft für Politik und Geschichte)

Adam, Jean-Michel. 1998. « Les genres du discours épistolaire », Siess, Jürgen (éd.). La lettre entre réel et fiction. (Paris : Sedes),pp. 37-53

Amossy, Ruth. 2006 [2000]. L'argumentation dans le discours (Paris : Colin)

Anscombre, Jean-Claude et Ducrot, Oswald. 1995 [1983]. L'argumentation dans la langue (Liège : Mardaga)

Callières, François de. 1717. De la manière de négocier avec les souverains, de l'utilité des négociations, du choix des Ambassadeurs et des Envoyez, et des qualitez nécessaires pour reüssir dans ces employs (Paris : M. Brunet). Rééd. Lempereur, Alain. 2002 (Paris : Droz). [trad. anglaise : 2000. On the Manner of Negotiating with Princes (New York : Houghton Mifflin)]

Kerbrat-Orecchioni, Catherine. 1998. «L'interaction épistolaire », Siess, Jürgen (éd.). La lettre entre réel et fiction (Paris : Sedes), pp. 15-36

Kerbrat-Orecchioni, Catherine.1998 [1e éd. : t. I, 1990, t. II, 1992, t. III, 1994]. Les interactions verbales (Paris : A. Colin)

Kerbrat-Orecchioni, Catherine. 1980. L'énonciation de la subjectivité dans le langage (Paris : Colin)

Kurbalija, J. and H. Slavik. 2001. Language and Diplomacy (Malta : Diplopublishing)

Nolke, Henning. 1993. Le regard du locuteur (Paris : Kimé)

Pascual, Edmond. 2004 [1989]. La communication écrite en diplomatie (Perpignan : Presses universitaires de Perpignan)

Renouvin, Pierre. 1955/1957. Histoire des relations internationales. Le XIXe siècle. De 1871 à 1914 : l'apogée de l'Europe (Paris : Hachette)

Siess, Jürgen (éd.). 1998. La lettre entre le réel et la fiction (Paris : Sedes)

Villar, Constanze. 2006. Le discours diplomatique (Paris : L'Harmattan)

Wicquefort, 1724. L'ambassadeur et ses fonctions (La Haye)

\section{ANNEXES}

Portrait de l'Empereur Guillaume II par l'ambassadeur Herbette dans son rapport du 14 juillet 1888

Hier, à Postdam, j'ai pu observer tout à loisir l'Empereur que je n'avais pas eu depuis plusieurs mois l'occasion de voir de près. Je l'ai trouvé très épaissi de carrure et, comme il n'est que de taille moyenne, la lourdeur du buste donne à l'ensemble de la personne une vulgarité disgracieuse. La tête est peu développée, le front n'a pas d'ampleur ; les 
pommettes sont saillantes ; les yeux clairs et sans expression. La physionomie générale est celle d'un sous-officier « bon enfant » mais dur et brutal.

[...] Au point de vue physique, l'empereur Guillaume qui n'a pas trente ans, a la force de la jeunesse et non la vigueur d'une robuste santé. Son sang doit être vicié par la scrofule, sinon par un principe cancéreux. Bien que sobre (je ne l'ai vu boire qu'un verre de vin du Rhin et manger que très peu), il a une tendance marquée à l'embonpoint. En un mot, il ne porte pas les signes de la longévité. Au point de vue moral, il ne me paraît pas d'une intelligence supérieure, son tempérament est vif, enjoué, brusque, audacieux et entreprenant. Tout cet ensemble ne m'inspire pas, je dois l'avouer, une grande confiance dans la modération et la sagesse du pupille de M. de Bismarck. (Berlin, 14 juillet 1888)

\section{NOTES}

1. Science critique née au XVIIe siècle (Dom Jean Mabillon, De re diplomatica libri sex, 1681), dont le but premier était de distinguer les faux qui proliféraient depuis le Moyen Age. Apparentée à la fois au droit et à l'histoire, la diplomatique étudie les actes (jugements, contrats... - ou diploma, appelés « chartres » au Moyen Age) datant de l'époque byzantine jusqu'aux « temps modernes ». 2. Signalons cependant le très récent ouvrage de $C$. Villar, parlant d'une " diplomaticité » pour définir ce discours diplomatique qu'elle considère comme un "type universel », situé "hors du temps et de l'espace » (Villar $2006: 9$ ).

3. Ce texte présente les conclusions d'une thèse de doctorat sur « Le discours diplomatique sous sa forme épistolaire: étude de la correspondance diplomatique sur les relations francoallemandes entre 1871 et 1914 », sous la direction de R. Amossy (décembre 2006, Université de Tel-Aviv).

4. Voici par exemple, un extrait d'une dépêche du 24 juillet 1911 :

«Votre Excellence ne partage pas mes appréhensions au sujet de la perspectives d'une nouvelle conférence pour régler les difficultés marocaines... je crois fermement que la réunion d'une conférence présente de graves inconvénients, et nous ôte tout espoir d'en finir avec les difficultés contre lesquelles nous luttons depuis longtemps... Je prie votre Excellence d'arrêter un moment son esprit sur les modifications apportées à l'état de choses existant au Maroc depuis 1906, du fait de l'Allemagne, de l'Espagne et de la France... Nous mettrons fin, il est vrai, en allant à la conférence, au dialogue que nous avons entamé avec l'Allemagne, et celle-ci aura l'affront de voir échouer les réclamations excessives qu'elle nous a présentées. Il se trouvera des Français pour qui cette satisfaction d'une minute suffira à masquer les conséquences graves de cette solution. Je ne pense pas que des hommes soucieux de l'intérêt de leur pays doivent se conduire par des considérations d'amour-propre... » (Dépêche du 24 juillet 1911 de l'ambassadeur Jules Cambon au ministre des Affaires étrangères de Selves)

5. Voir par exemple cette dépêche du président Thiers adressée à Gontaut-Biron, premier ambassadeur de France à Berlin après la guerre de 1870 :

« Nous voulons la paix, nous devons la vouloir pour notre sûreté intérieure autant que pour notre sûreté extérieure. Le contraire serait de notre part, de la folie. A mon âge, je ne puis désirer d'autre gloire, si je puis aspirer à en avoir, que celle de pacifier mon pays, de lui procurer quelques années de repos, de calme, de bien-être et de lui procurer, en un mot, non pas du bruit mais du bonheur. C'est le bonheur qui lui rendra des forces et qui remettra son moral. J'ai vaincu la démagogie par le canon; je ne vaincrai l'anarchie intellectuelle et morale que par un long apaisement. Je comprends cela, et, si je ne le comprenais pas, je n'aurais pas signé la paix que j'ai 
signée, le cœur déchiré, mais l'âme haute, parce que je savais qu'il n'y avait pas autre chose à faire. » (Dépêche du 18 avril 1872)

6. Autres exemples:

«Bien cher ami, Je reçois votre lettre et votre dépêche du 14. Je m'associe à vos sentiments et à vos impressions. Je les partage. Je vous remercie et je vous embrasse avec reconnaissance » (Dépêche du 16 mai 1875 du duc Decazes, ministre des Affaires étrangères à l'ambassadeur Gontaut-Biron).

«Je n'ai jamais cru pour ma part, à la possibilité d'un concours effectif et sincère de l'Allemagne dans une négociation quelconque pouvant tourner au profit de la France. Vous devinez sans peine que les derniers événements ne sont pas de nature à me faire revenir sur mes sentiments antérieurs. " (Lettre particulière du ministre Flourens à l'ambassadeur Herbette, sans date d'expédition, arrivée à l'ambassade le 16 mars 1887)

«J'ai plus d'une fois entretenu vos prédécesseurs des sentiments pacifiques de l'Empereur, mais je leur ai en même temps indiqué que le souverain ne serait peut-être pas toujours de force à opposer une résistance victorieuse à certains courants qui peuvent entraver la paix. » (Dépêche du 22 juin 1914 de l'ambassadeur Jules Cambon)

7. Exemple tiré des entretiens d'avril 1911 entre Jules Cambon et Kiderlen-Wächter sur une éventuelle occupation française de Rabat et la possibilité d'une action française sur Fez. Dans une lettre du 5 avril 1911 Jules Cambon avait tenté de persuader le secrétaire d'État allemand de la nécessité pour la France d'occuper le port de Rabat, afin d'assurer la sécurité des Européens résidant à Fez. L'ambassadeur y affirme entre autre que la France n'a pas l'intention par son action de modifier le statu quo instauré par l'accord d'Algésiras de 1906, qui consacrait l'indépendance du Maroc. Dans sa réponse, le secrétaire d'État allemand reformule les propos de son interlocuteur en soulignant le caractère temporaire (mot non employé dans la lettre française) de l'occupation éventuelle de Rabat pour le mettre en doute, en se fondant sur un précédent (l'occupation française de Casablanca, laquelle, intervenue dans des circonstances semblables, est devenue permanente) et introduire son propre discours.

8. Il s'agit de la question de la libération des prisonniers après la guerre de 1870 .

9. Les dépêches fourmillent alors de réflexions de ce type :

"J'ai pu me convaincre malheureusement une fois de plus que, grammaticalement et moralement parlant, le mot français de générosité n'avait pas son équivalent dans la langue allemande [...] » (Dépêche du 22 novembre 1871, de MM. Goulard et de Clerq, plénipotentiaires français aux négociations de Francfort).

« Ne voyez-vous pas déjà poindre cette tactique favorite du Prince de Bismarck, consistant à représenter ses adversaires comme les agresseurs, et l'Allemagne obligée bien malgré elle de faire une guerre qui n'est qu'un acte de défense?» (Dépêche du 7 mai 1875 de l'ambassadeur Gontaut-Biron au duc Decazes)

10. Au Marquis de Noailles qui lui vante en 1899 les vertus du rapprochement avec l'Allemagne, Maurice Paléologue, alors directeur de cabinet au ministère des Affaires étrangères, objecte l'Alsace-Lorraine, et Noailles capitule : «Je n'ai plus rien à dire... Je ne suis plus compétent: l'Alsace-Lorraine, ce n'est plus de la diplomatie!» 


\section{RÉSUMÉS}

Cette étude décrit les caractéristiques du discours diplomatique qui s'est tenu entre la France et l'Allemagne de 1870 à 1914, à travers l'analyse de deux «sous-genres » de la correspondance diplomatique : les lettres échangées entre le ministère et les ambassadeurs, et la correspondance interétatique. Ces deux genres relèvent de pratiques socio-discursives différentes et ne poursuivent pas les mêmes objectifs. Leur point commun est néanmoins le rôle central joué par l'ambassadeur qui est l'axe de la communication. Bien que très formelles et distanciées en vue de préserver l'harmonie internationale, les notes entre États sont des textes fortement argumentatifs qui cristallisent des positions antagonistes et expriment des points de vue subjectifs «masqués» par des arguments rationnels. Les rapports diplomatiques, en dépit de leurs contraintes génériques, comportent des enjeux relationnels et laissent un espace considérable à la présentation de soi, ou construction d'ethos. L'analyse de leur spécificité permet à la fois d'éclairer le fonctionnement de l'interaction discursive entre les deux pays au long de la période, et de dégager les différents aspects d'un discours jusque-là relativement ignoré par les sciences du langage. Au-delà de la distinction entre les genres, certains traits constitutifs du discours diplomatique peuvent être dégagés. Dans l'obligation de concilier des objectifs antithétiques tels que la défense des intérêts nationaux et la préservation de la paix internationale, le discours diplomatique a recours à des formes linguistiques qui privilégient l'indirection et l'implicite. Il a également recours à des formes d'échange ritualisées permettant de sauvegarder le territoire et la face des nations. Les frontières identitaires entre les egos nationaux transparaissent également dans la matérialité du discours, en particulier dans les périodes de crise internationale.

This paper describes the characteristics of the diplomatic discourse between France and Germany from 1870 to the First World War through the analysis of a corpus of diplomatic correspondence. The latter is divided into two main genres: the letters exchanged between foreign ministries and ambassadors, and the correspondence between states. These two genres reflect different socio-discursive practices and aim at different objectives; their main common point is the role played by the ambassador as the central axis of the communication. Although very formal and distanced in order to preserve international harmony, the notes between states are strongly argumentative texts which crystallize antagonist positions and express subjective points of view "masked" by rational arguments. Diplomatic reports, in spite of their generic constraints, have high relational stakes, and leave considerable room to self-presentation, or the construction of ethos. The concomitant analysis of these two forms of diplomatic correspondence enlightens the way diplomatic exchanges work at this period and helps to understand the evolution of the discursive interaction between the two involved states. Beyond this distinction between genres, some constitutive features of diplomatic discourse can be identified. Because it must reconcile antithetical aims such as defense of national interests and preservation of international peace, diplomatic discourse has to use linguistic forms that are oblique and implicit. It also recurs to ritualized exchanges in order to save the territory and the "face" of nations. Identity borders between national egos can be perceived as well in the materiality of the discourse, especially during periods of international crisis. 
INDEX

Mots-clés : discours, échange épistolaire, genre, diplomatie

Keywords : discourse, epistolary correspondence, genre, diplomacy

\section{AUTEUR}

\section{SIVAN COHEN-WIESENFELD}

Université de Tel-Aviv, ADARR 